\title{
Performance Evaluation of RPL Routes and DODAG Construction for IoTs
}

\author{
Khalid Mohamed ${ }^{1}$, Suleiman $\mathrm{Ali}^{2}$, Soilahoudini Ali ${ }^{2}$, Ilyas Kassim² \\ Department of Computer Engineering, Al-Neelain University ${ }^{l}$ \\ Department of Electrical and Electronics Engineering, International University of Africa ${ }^{2}$ \\ Sudan
}

\begin{abstract}
The rapid growth in the Internet of Things (IoTs) has change our life to be more intelligent and smart, the development in the Wireless Sensors Networks (WSNs), besides the wide use of the embedded devices in different area like industry, home automation, transport, agriculture and health care, which was led the Routing Over Low-power and Lossy-network (ROLL) working group to introduce the IPv6 Routing Protocol for Low-Power and Lossy Networks (RPL), therein the RPL nodes have organized topology as a Directed Acyclic Graph (DAG) and terminated at one root to form the Destination Oriented DAGs (DODAGs). In this paper by using InstantContiki3.0 and CoojaGUI we analyze the DODAG formations, the RPL control messages that are send downward and upward routes to construct and maintain DODAG and the Rank computation by Objective Function (OF) for inconsistency and loop detection, also we evaluate the performance of the RPL based on the Expected Transmission Count (ETX) OF that enable RPL to select and optimize routes within RPL instance, as well as we evaluate the following metrics: The ETX Reliability Object (ETX), Radio Duty Cycle $(R D C)$, energy consumption, the received packets by the motes and neighbor count. The simulation results show that the RPL control messages flow in consistent manner, the DODAG root able to connect to all of the neighbor motes, also Rank illustration shows no loops and DODAG topology consistent, as well as the ETX can essentially take control over DODAG formations and it has an effects in the RDC ratio, furthermore most of the motes show reasonable low power consumption, also the motes show acceptable number of the received packets.
\end{abstract}

Keywords: RPL, DODAGs, DIO, ETX, OF, RDC, Rank, Sensor, IoT

\section{Introduction}

The IPv6 Routing Protocol for Low-Power and Lossy Networks (RPL) use to support the traffic flow in the Low-powered Lossy Networks (LLNs), that include point-to-point connection between devices (inside the LLN), point - to - multipoint connection (from central control point to a subset of devices inside the LLN) and multipoint-to-point connection (from devices inside the LLN towards a central control point).

LLNs are constrained network typically the nodes or the routers are operating with constrains condition with limited processing power, memory and energy, in addition to the interconnect links are lossy, so, it can support only low data rates that are usually unstable with the relatively low data packet delivery rates, however, it may potentially consist of few dozen to thousands of routers. LLNs, such as Radio Networks, do not typically have predefined topologies, however, RPL routes are optimized for traffic to or from one or more roots therefore a node within the DAG that has no outgoing edge act as sinks for the topology.

RPL organizes a topology as a Directed Acyclic Graph (DAG) a directed graph having the property all edges are contained in paths oriented toward and terminating at one or more root nodes, that is partitioned into one or more DODAGs, in other words one DODAG per sink (a DAG rooted at a single destination) [4].

Low-power wireless devices present difficult constraints, however, routing over such low power and lossy networks introduce requirements that existing routing protocols such as Link State Protocols (OSPF, IS-IS and OLSR) and Distance Vector protocols (RIP, AODV and DSR) may not fully adequate in LLNs, therefore the ROLL [7] working group drive five criteria (routing state, loss response, control cost, link cost and node cost) to satisfy routing in low power and lossy networks. None of the existing Internet Engineering Task Force (IETF) routing protocol specification meets these criteria, therefore, the need for having a routing protocol for LLNs requires new protocol specification documents.

The industrial IoT for LLNs will significantly improve the productivity and safety of industrial plants, however, the wireless LLN nodes in the industrial environments have low-power and low-cost devices that have limited memory, [12] analysed the requirements for a routing protocol must be used, so the routing algorithm must support the node constraints include power and memory as well as after 
adding new device determine how to rout the packets in the network.

The home-control automation that may include a high number of wired and ad hoc devices e.g. actuators, sensors, and advanced controllers, [13] specifies the routing requirement for constrained devices, therefore the routing is required because ROLL nodes only cover a limited radio rang and routing protocol must support constraint-base routing.

The new version of the Internet Protocol (IP) version 6 (IPv6) [11] is introduced as the successor to IP version 4 (IPv4), so ipv6 expanded addressing capabilities from 32 bits to 128 bits to support more levels of addressing, increase number of addressable devices and simpler auto-configuration of addresses.

IEEE Std 802.15.4 [16] define short-range Radio Frequency (RF) transmissions in a Wireless Personal Area Network (WPAN) to enabled very low-cost, low-power communications, it has provide some capabilities such as Carrier Sense Multiple Access with collision avoidance (CSMA-CA) or ALOHA for a channel access, low power consumption, fully acknowledged protocol for transfer reliability and unique 64-bit extended address or allocated 16-bit short address, as well as it operates in either of two topologies: the star topology or the peer-to-peer topology.

In the star topology, the communication is established between devices and a single central controller (PAN coordinator), the peer-to-peer topology also has a PAN coordinator; however, it differs from the star topology in that any device is able to communicate with any other device as long as they are in range of one another, therefore, many applications can benefit from the peer-to-peer topology such as industrial control and monitoring, wireless sensor networks, asset and inventory tracking, intelligent agriculture, and security; as well as allows multiple hops to route messages from any device to any other device on the network.

LLNs are made up of many embedded devices with limited power, memory, and processing resources, they are relies on a variety of links, such as IEEE 802.15.4, in which the frame size is limited to 127 bytes per frame, therefore, it's much smaller values than the guaranteed Maximum Transmission Unit (MTU) of IPv6 1280 bytes, for this reason the need to compress IPv6 packets over IEEE 802.15.4 led to the writing of Compression Format for IPv6 Datagram's over IEEE 802.15.4-Based Networks [14].

Thubert et al. [15] introduces a new specification to IPv6 over Low-Power Wireless Personal Area Network (6LoWPAN) dispatch type for use in 6LoWPAN route-over topologies, which initially covers the needs of Routing Protocol for Low-Power and Lossy Networks (RPL) data packet compression by using this dispatch type, furthermore, defines a method to compress the RPL Option information and Routing Header type 3 (RH3). It also introduces a new 6LoWPAN Routing Header (6LoRH) to carry
IPv6 routing information. The 6LoRH may contain source routing information such as a compressed form of a Source Routing Header (SRH), as well as other sorts of routing information such as the RPL Packet Information (RPI) and IP-in-IP encapsulation within the LLN.

IPv6 over low power Wireless Personal Area network (6LoWPAN) adaptation layer was introduced to carry IPv6 datagram over constrained links, [17] provides intensive analysis of 6LoWPAN specifications that includes IPv6 encapsulation, frame format, 6LoWPAN header compression, fragmentation of the payload datagram and encoding of User Datagram Protocol (UDP).

In the 6LoWPAN header compression the IPv6 can be compressed to two octets instead of 40 octets, one octet use for $\mathrm{HC} 1$ encoding and the other one use for the Hop Limit, and the next header field is UDP, Internet Control Message Protocol (ICMP) or Transmission Control Protocol (TCP); fragmentation of the payload datagram: The fragmentation header add to the payload datagram when the datagram does not fit within a single IEEE 802.15.4 frame as a result it broken into link fragments, however if it fit within a single IEEE 802.15.4 frame it's not fragmented and encapsulation not contain fragmentation header; and encoding of user datagram protocol UDP: the UDP header it compressed from the original size 8 octets to 4 octets.

IoT usually implemented over UDP [22] instead of TCP [21] because header size of the TCP 20-byte header and UDP 8-byte header as well as a connection setup ,the flow and congestion control, overhead and buffering in TCP, in spite of TCP provides a connection-oriented, end-to-end reliable, securable connection service between pairs of processes, also its able to transfer a continuous stream of octets in each direction as shown in [18].

RPL requirements such as reliability, mobility, scalability which are a very important characteristic of a routing protocol, in addition it will have a direct impact on network reliability and performance analyzed in [19] and highlighted other challenges that emerged since it is defined as a standard as well as address three types of traffic patterns, also compares the RPL implementations for TinyOS and Contiki, the most popular operating systems for low-power devices.

Power consumption in the low power wireless devices is important to maintain a long network lifetime, the low-power radio transceivers consume too much power when passively listening for radio traffic, as well as the power consumption when transmitting radio data is slightly higher [1][23], therefore the duty cycle mechanism must be implemented to turn the transceiver on and off to save the power.

The radio duty cycle protocols use the time scheduling protocols asynchronous and synchronous protocols[24], in synchronous duty cycle the nodes 
synchronize their wake-up periods while asynchronous duty cycle let every node free to determine it is own independent sleep schedule, therefore asynchronous duty cycle protocols are well suit to low bandwidth network because they are less complex to implement and particularly energy efficient.

The Contiki operating system and COOJA simulator were used in [8] to performs an in-depth study on implementation of the RPL, to provide insights and guidelines for the adoption of these standards, specifically, to evaluate the behaviour of the ContikiRPL implementation and perform analysis to the performance of network construction process by measuring several important metrics; as well as provided an investigation of the performance and possible situations during the functioning stage of the WSN indicate that RPL is a pretty robust protocol for WSN.

[20] presents the standards proposed by the ROLL working group (WG) RPL and DODAG graph to route data traffic, it also reviews the lack of the current IETF routing protocol to provide acceptable performance in LLNs against the following metrics: scalability, packet loss, control overhead and ability to impose routing constraint.

RPL based on graph construction directed acyclic graph (DAG) to form Destination-Oriented DAG (DODAG). The Rank of a node is a scalar representation of the location of that node within a DODAG Version, therefore the Rank of the nodes decrease as the DODAG Version is followed towards the DODAG destination, as a consequence the Rank is used to avoid and detect loops, moreover the Rank value feeds into DODAG parent selection, according to the RPL loop-avoidance strategy.

The stability of the Rank determines the stability of the routing topology, the Rank does not necessarily change as fast as some link or node metrics would. Besides the Rank is an abstract numeric value, but it is the expression of a relative position within a DODAG Version with regard to neighbours, and it is not necessarily a good indication or a proper expression of a distance or a path cost to the root. Additionally, the Rank is not a path cost, however it can be derived by the Objective Function, the computation may depend on, e.g. parents, link metrics, node metrics, and the node configuration and policies.

RPL use data packet for on demand loop detection, so, the RPL packet information that is transported with the data packet includes the Rank of the transmitter, whenever there is an inconsistence between the routing decision for a packet and Rank e.g. a packet flagged as moving in the upward direction and the packet information records the Rank of the transmitter is lower than the Rank of the receiving node, as a result the receiving node is able to conclude there is a possible loop, as well as that DODAG is inconsistent and a node institutes a local repair operation [4].
The rest of the paper is organized as follows. In section II we present comprehensive analysis of RPL control message ICMPv6 and DODAG formations, Rank computation and routing metric as metric or constraint. Section III provides a general overview of the RPL routing environment for the two study scenarios and simulations topology. In section IV we evaluate the simulations results and discuss the DODAG construction, Rank computation and routing metric. Finally, section V concludes this paper.

\section{Comprehensive Analysis of Rpland Dodag}

\subsection{RPL Control Message ICMPv6}

The RPL use ICMPv6 control message to send the source address that is a link-local address, and destination address that can be a link-local uncast address of the destination or multicast address of all RPL nods, this multicast address is a new address with a value of ff02::1a.

i. DODAG Information Solicitation (DIS): The DODAG Information Solicitation (DIS) use by the node to probe or solicit it is neighbourhood for nearby DODAGs, however, it is analogous to IPv6 Router Solicitation; therefore, it is used to solicit a DODAG information object from RPL nodes.

ii. DODAG Information Object (DIO): The RPL provisions routes up toward DODAG roots through DODAG Information Object (DIO) that allow a node to discover the RPL instance according to an Objective Function (OF) as well as to know it is parent configuration. Furthermore, it uses in the selecting of a DODAG parent set and maintain the DODAG.

ii. Destination Advertisement Object (DAO): RPL constructs and maintains downward routes with the Destination Advertisement Object (DAO), in the storing mode the DAO message is uncast by the child to the selected parent(s), downward routes also support P2MP flows from the DODAG roots toward the leaves [4].

The Figure 1 illustrates the Destination Oriented Directed Acyclic Graph (DODAG) formations in addition to DIS, DIO and DAO messages flow, node 2 and node 3 lay within the radio range of node 1(DODAG root) and node 4 lays out the radio range of the root, it can connects only through the node 2 or node 3 to the root, whenever node 4 sent DIS to node 3 and node 2 to join the DODAG, they are respond by DIO messages, as a consequence if node 4 e.g. selects node 2 as its preferred parent according to ETX calculation and decide to join DODAG, in NonStoring mode DAO message is uncast to the DODAG root (node 1) as it has shown by the dash arrow and it is ignored by the other nodes other than DODAG root. 
However, in Storing mode the DAO message is uncast by node 4 to the selected parent (node 2 ) as a result it provisions node 2 as next-hop for the default route and advertising the ETX, in the figure the solid arrow shows the DODAG routes (connection links) and the dot lines show the RPL Control Messages.

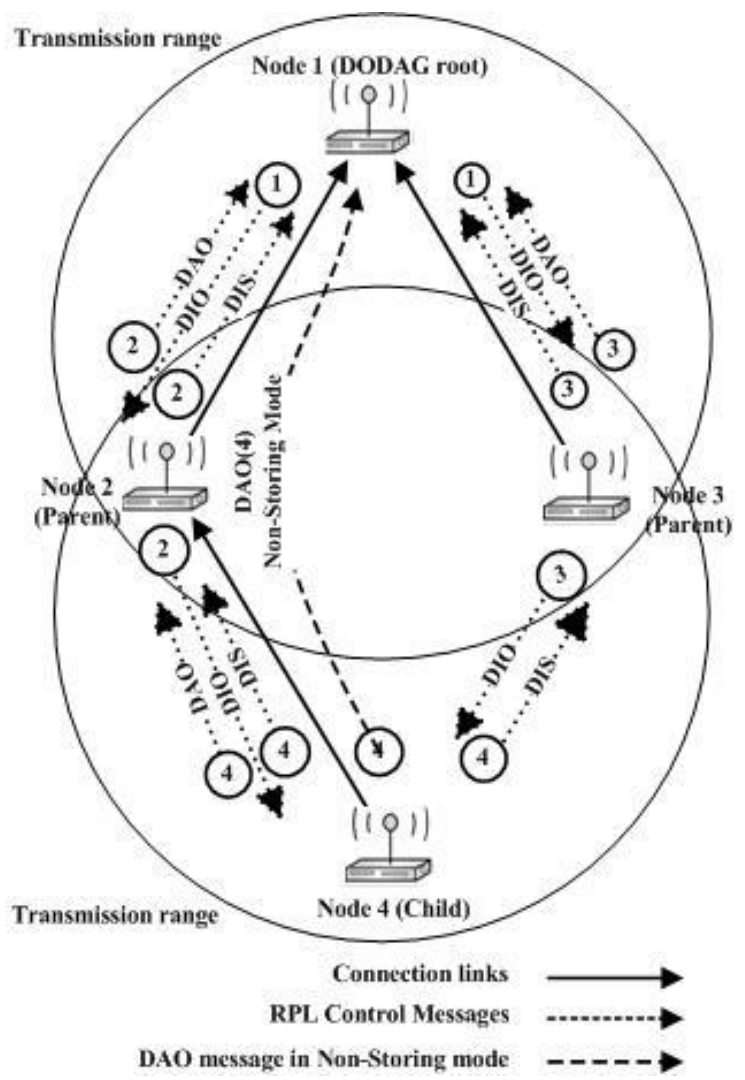

Figure 1. DODAG formations

The DODAG Information Solicitation (DIS) message trigger DIO message, RPL use DODAG Information Object (DIO) message to discovers and maintains upward routes, RPL use Destination Advertisement Object (DAO) message to constructs and maintains downward routes, so to establish downward routes RPL send DAO message upward.

\subsection{The Trickle Algorithm}

RPL implement the trickle timers [5] to govern the sending of the DIO message, therefore the trickle algorithm controls the sending rate and let it to stay consistent, because it allows the nodes in LLNs to exchange information in a highly robust, energy efficient, simple and scalable manner.

\subsection{Objective Function Zero (OF0)}

The RPL specification describes constraints on how nodes select potential parents, called a parent set, from their neighbours, hence all parents are feasible successors for upward traffic, however, a RPL OF states the outcome of the process used by a RPL node to select and optimize routes within a RPL instance based on the information objects available.

The goal of the OF0 is for a node to join a DODAG version that offers good enough connectivity to a specific set of nodes, thereby, objective Function Zero is designed to selects a preferred parent and a backup feasible successor parent if one is available, and fined the nearest grounded root [9].

\subsection{The Minimum Rank with Hysteresis Objective Function (MRHOF)}

Minimum Rank with Hysteresis Objective Function (MRHOF), an Objective Function (OF) for RPL, which uses hysteresis while selecting the path with the smallest metric value this metric is determined by the metrics in the DIO Metric Container to preventing excessive churn in the network, however, MRHOF seeks to minimize path costs whenever it uses with the Expected Transmission Count (ETX) metric it will allows RPL to find the stable minimumETX paths from the nodes to a root in the DAG instance, also with the latency metric allows RPL to find stable minimum-latency paths from the nodes to a root in the Directed Acyclic Graph (DAG) instance [10].

\subsection{Computing the Rank}

DAG roots set their Rank to MinHopRankIncrease [10]. An OF0 implementation first computes a variable step-of-rank which it is strictly positive integer and an intermediate computation based on the link properties with a certain neighbor. Computing a step-of-rank based on a static metric such as an administrative cost implies that the OF0 implementation only considers parents with good enough connectivity, and results in a Rank that is analogous to the hop-count.

An implementation should allow the operator to configure a factor called rank-factor and to apply the factor on all links and peers to multiply the effect of the stretched step-of-rank in the rank-increase computation, rank-increase it is strictly positive integer which it is delta between the Rank of the preferred parent and self.

The step-of-rank $(\mathrm{Sp})$ that is computed for that link is multiplied by the rank-factor (Rf) and then possibly stretched by a term ( $\mathrm{Sr}$ ) that is less than or equal to the configured stretch-of-rank. The resulting rankincrease ( $\mathrm{Ri}$ ) is added to the Rank of preferred parent $(\mathrm{R}(\mathrm{P}))$ to obtain the Rank of this node $(\mathrm{R}(\mathrm{N}))$ [9]:

$$
R(N)=R(P)+R i
$$

Where: 
$R i=(R f \times S p+S r)$ MinHopRankIncrease

The Objective Function (OF) operates on the entire (i.e., 16-bit) Rank quantity; the integer portion of the Rank can be used for determination of parent relationships or loop detection, so the integer portion of the Rank is computed by the DAGRank() macro as follows:

DAGRank $($ rank $)=$ floor $\left(\frac{\text { rank }}{\text { MinHopRankIncrease }}\right)$

Where floor( $\mathrm{x})$ is the function that evaluates to the greatest integer less than or equal to $\mathrm{x}$.

\subsection{Routing Metric}

Routing in Low-power and Lossy Networks (LLNs) require specific routing characteristics in contrast with the traditional wired and ad hoc networks, therefore the specific set of the link and node routing metric and constraints suitable for building automation home and industrial routing requirement are built according to the objective function.

i. The ETX Reliability Object (ETX): The ETX provide discrete value as the number of transmission a node expects to make in order to successfully deliver a packet to destination, however, it may be used as a constraint or path metric.

- ETX Constraint : The ETX object common header indicates that the value related to a constraint, in this case the ETX must not exceed this value.

- $\quad$ ETX metric: The value of the ETX is updated along the path to reflect the path quality, whenever a node selects any node as its preferred parent the node update the path ETX and advertising itself the new path ETX.

The cumulative path ETX is the sum of the link ETX of all of the traversed links from the advertising node to the DAG root [6]. So the ETX provides a discrete value may compute by the following formula:

$$
E T X=\frac{1}{(D f \times D r)}
$$

Where Df is the measured probability that a packet is received by the neighbor and Dr is the measured probability that the acknowledgment packet is successfully received. ii. Radio Duty Cycle (RDC): radio duty cycle mechanism used to control the radio transceiver (on, off), the wireless transceiver often has the highest power consumption [23], so the RDC uses periodical wake up to listen for packet transmission from the neighbours.

iii. Power: power and energy are critical resources in LLNs, therefore the node energy may be used as a metric or a constraint [6], in constraint based the routing protocol may avoid the selection of the node with low residual energy as a result it may computes a longer path in order to increase the network life duration.

\section{RPL Routing Environment and Simulation}

We evaluate the performance of the IPv6 Routing Protocol for Low-Power and Lossy Networks (RPL), DODAG construction and Rank consistency based on InstantContiki3.0 and CoojaGUI virtual platform to run contiki, which is an open source operating system for the internet of things applications [1], for sensors devices we utilized the Tmote Sky [3] with MSP430bored platforms that is an ultra low powered IEEE 802.15.4 compliant module for use in sensor networks which can enables a wide range of mesh network application [2], at network layer we used RPL to support the traffic inside the LLNs as well as Uipv6 stack to provides IPv6 networking, furthermore for adaptation process 6LowPAN it used to perform compression and fragmentation for the IPv6 and UDP packets with the maximum transmission unit(MUT) of 127 byte, in RDC layer we used a low power listening based protocol ContikiMAC which it provides a very high power efficiency that it is implemented according to the IEEE 802.15.4 specification, Table I. below summarize the simulation parameters values for the two scenarios.

The Figure 2 shows the simulation topology for the first scenario, mote number one act as RPL BorderRouter (root) to bridge the sensors network to the rest of the internet, the Wireshark[25] used to overheard and sniffs the radio packets, consequently the different DODAG frames messages captured for further analysis of the RPL Control Messages (DIS, DIO and DAO) construct the DODAG and the Rank.

The mote received the DIS message it transmits a DIO message using a trickle timer, therefore for consistency the sender Rank must be less than the receiver Rank otherwise it considers inconsistent and case the trickle timer to reset, the DODAG root mote number one has stored the source routing information learned from DAOs in order to construct the source routes. 


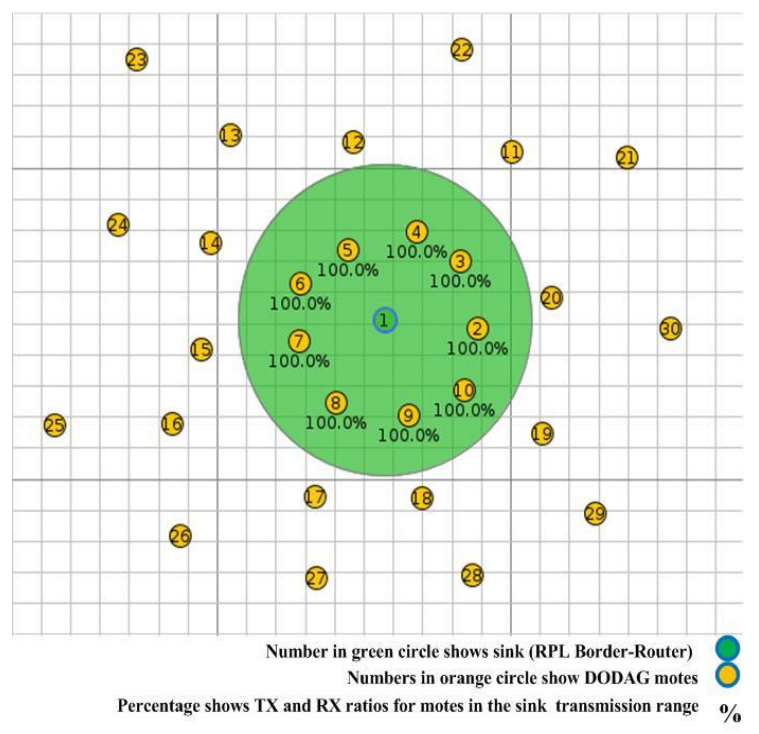

Figure 2. RPL routing environment for the first scenario sink act as Border-Router

The Figure 3 shows the simulation topology for the second scenario, the simulation starts with an unpredictable environment, so the motes advertise their presence by sending DODAG Information Solicitation (DIS) messages to solicit neighbor and trigger DIO transmissions to join the DODAG, as well as sending of DAO messages to construct or maintain existing DODAG.

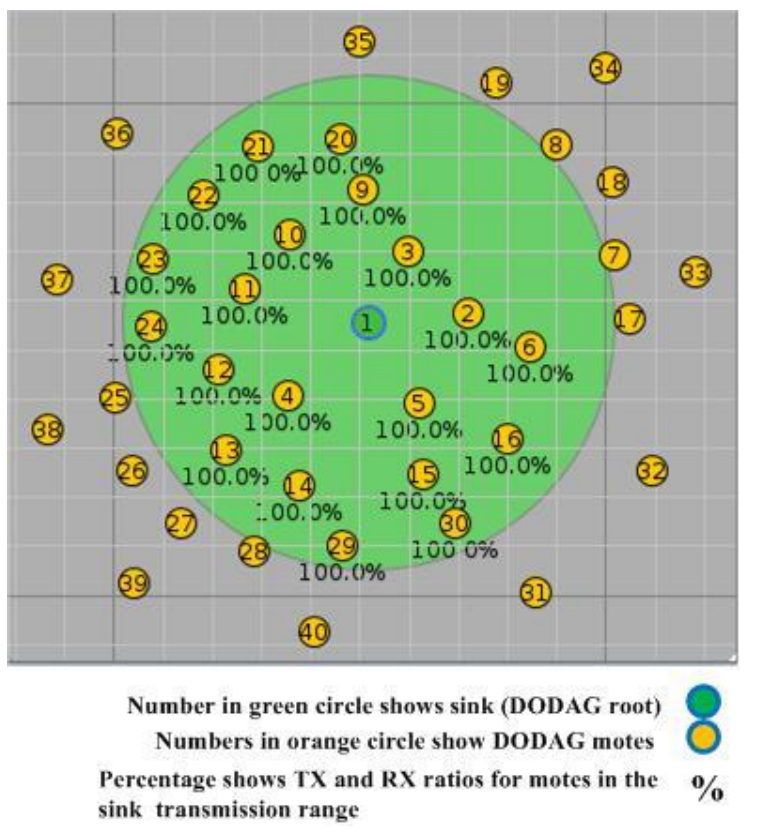

Figure 3. RPL routing environment for the second scenario

To characterize the various capability of the RPL protocol the motes are arrange in different transmission rang from the sink mote, we apply $10 \mathrm{~m}$ background grid to show the distances of the motes, as they have shown in Figure 3 most of the motes lay within the transmission range $50 \mathrm{~m}$ and Interference range $100 \mathrm{~m}$, so motes numbers $7,8,17,18,19,25,26$, 27,28 they are adjacent to the transmission rang and the far away motes like 33, 34, 38, 39, 40 can establish the connection with sink only via tow traverse routers (motes) or more depend on ETX calculations.

Table 1. Simulation Parameters

\begin{tabular}{|l|l|c|}
\hline \multicolumn{1}{|c|}{ Parameters } & \multicolumn{2}{c|}{ Values } \\
\hline Scenarios & First scenario & $\begin{array}{c}\text { Second } \\
\text { scenario }\end{array}$ \\
\hline Numbers of Motes & 30 & 40 \\
\hline Sensor Type & Tmote Sky \\
\hline Transport layer & UDP \\
\hline Network layer & Uip6, RPL \\
\hline Adaptation & 6LowPAN \\
\hline MAC device & CSMA/CA \\
\hline RDC & contikiMAC \\
\hline Physical & IEEE802.15.4 \\
\hline $\begin{array}{l}\text { Radio } \\
\text { Transmission and } \\
\text { Interference range }\end{array}$ & 50m,100m \\
\hline Channel Model & UDGM \\
\hline Simulation Time & 1,200seconds & 1,500 seconds \\
\hline
\end{tabular}

\section{Performance Evaluation}

\subsection{First scenario}

The Figure 4 represents DODAG Information Solicitation (DIS) messages which it described by the frame numbers and the time of sending each DIS frame from the start time of the simulation (when the motes powered up) until the end of the simulation, but all the DIS message were sent in the time interval between start time to time equal 50 second, so the motes send multiple DIS messages as an initial probe to their neighbour for nearby DODAGs and to solicit a DODAG Information Object (DIO) messages from RPL motes.

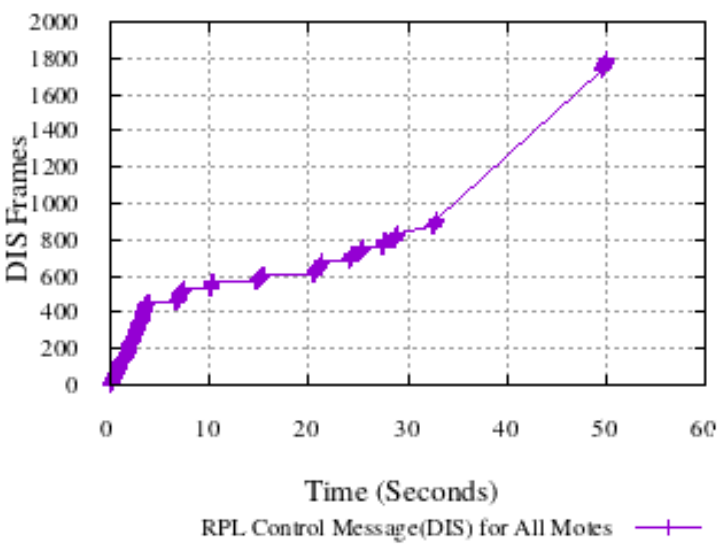

Figure 4. DODAG Information Solicitation (DIS) frames versus time

The Figure 5 represents the relationship between a DODAG Information Object (DIO) messages and the time, this DIO messages have triggered by the DIS messages to discover the RPL instance start at such 
time between 30 and 50 seconds differ from motes located in the root radio range to another motes that are located outside the root radio range, however the sending of this DIO message is controlled by the trickle timers to control the sending rate of the DIO messages periodically and to stay consistent.

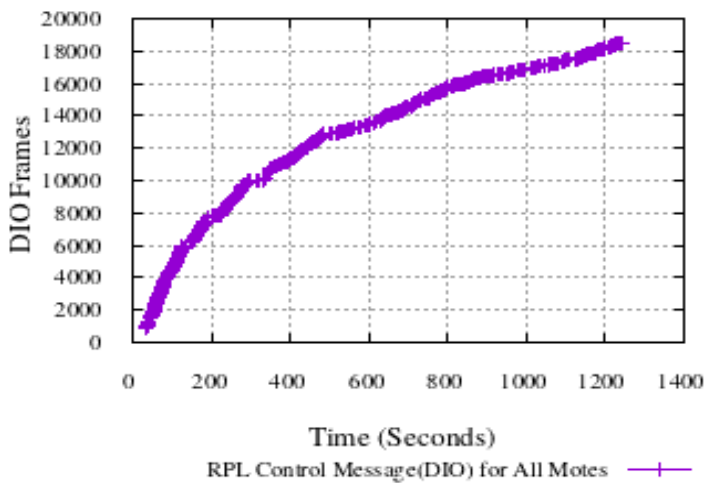

Figure 5. Destination information Object (DIO) frames versus time

As a result, the motes listen to the DIO messages and use their information to join this new DODAG network as well as selecting DODAG parent(s) and provision one of this parent as the next hop for the default route, furthermore the motes use the Rank values which is carrying in the DIO messages to update and maintain the DODAG, to avoid the loop and for inconsistency detection.

The Figure 6 shows Destination Advertisement Object (DAO) messages versus the time for the motes started at time 35 second, after the motes issued the DIS messages and received the DIO messages to construct and maintain the down route, because the mote can trigger their sub-DODAG to send the DAO messages by using the DAO Trigger Sequence Number (DTSN) through the DIO message, also the DAO flow upward the DODAG when a mote received a unicast DAO can trigger sending a unicast DAO to a DAO parent.

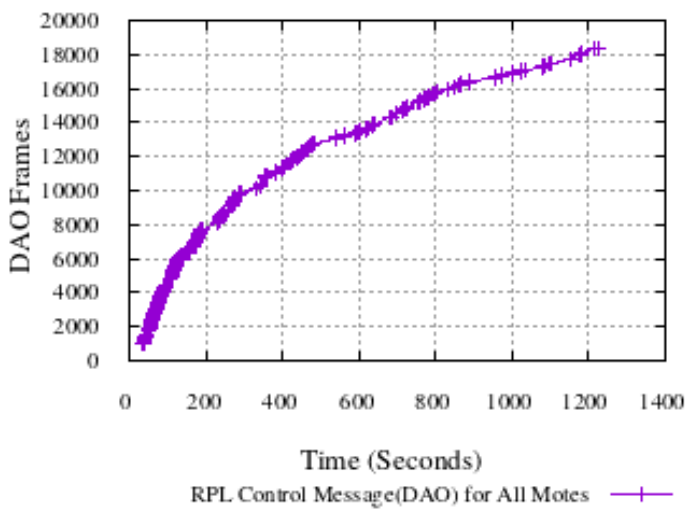

Figure 6. Destination Advertisement Object (DAO) frames versus time

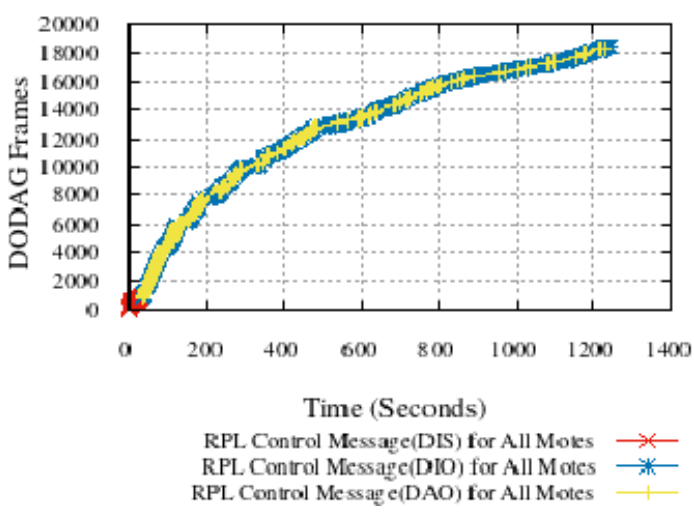

Figure 7. Destination-Oriented Directed Acyclic Graph (DODAG) frames

The Figure 7 depicts the sequence of the transmission of the DODAG messages (DIS, DIO and DAO) to figure out the construction of the DODAG topology so at the start time the motes send about 913 DIS messages to solicits the information, afterwards the root motes advertises the Ranks values and the motes send about 9860 DIO messages periodically which they are controlled by the trickle timers to discover and maintain the upward routes, and then the motes issue about 6575 DAO messages which it scheduled by the Delay DAO timer, so these messages create Destination-Oriented directed acyclic graph to construct and maintain the downward routes, as we can see most of the RPL Control Message it is DODAG Information Object (DIO) messages and DODAG Information Solicitation (DIS) messages issue only at the initial period to probe the neighbour motes.

The Rank determines the stability of the routing topology and feeds into DODAG parent selection, therefore inconsistence between the routing decision for a packet and Rank between the two motes indicates a possible loop.

The Figure 8 below depicts the Rank values for the 30 motes with respect to the time, since the start time the DODAG root advertises a fixed Rank value equal to 250 as a MinHopRankIncrease, the other motes according to the Objective Function (which it designed to find the nearest grounded root) in time interval between start time 0 until 100 seconds advertise their initial Ranks in response to the DIO messages from the root, these Ranks have high value increase in the downward direction as illustrate in the figure e.g. the mote number 9 inside the radio transmission range of the mote number one has Rank value equal to 750 , the adjacent motes to radio transmission range like mote number 15 has Rank value equal to 1250 and the motes lay far away the from radio transmission range like motes number 24 and 25 have Ranks value more than 1750 and 2000 respectively. However, we observe the motes use the Objective Function zero (OF0) to optimize Rank values in consistent manner, so e.g., the mote number 
9 lays inside the radio range have Rank value decreased to become 500, the mote number 15 has Rank value decreased to become 750 and the mote outside the radio range number 24 and 25 have Rank value decreased to become 1000 and 1250 respectively, in this context all other motes use the OF0 to optimize their Rank values. Besides OF0 scans all candidate neighbour to finds the best parent from the parent set and the motes that provide good connectivity.
Consequently, the motes with the progress in the simulation time select the preferred parent that will cause the lesser resulting Rank.

Moreover, we observe from Figure 2 and Figure 8 the motes inside the radio transmission range of the root have less Ranks values than the adjacent motes to the radio transmission range, and the adjacent motes have less Ranks values than the motes lay far away from the radio transmission range as a result there is no probability that loops will be occur.

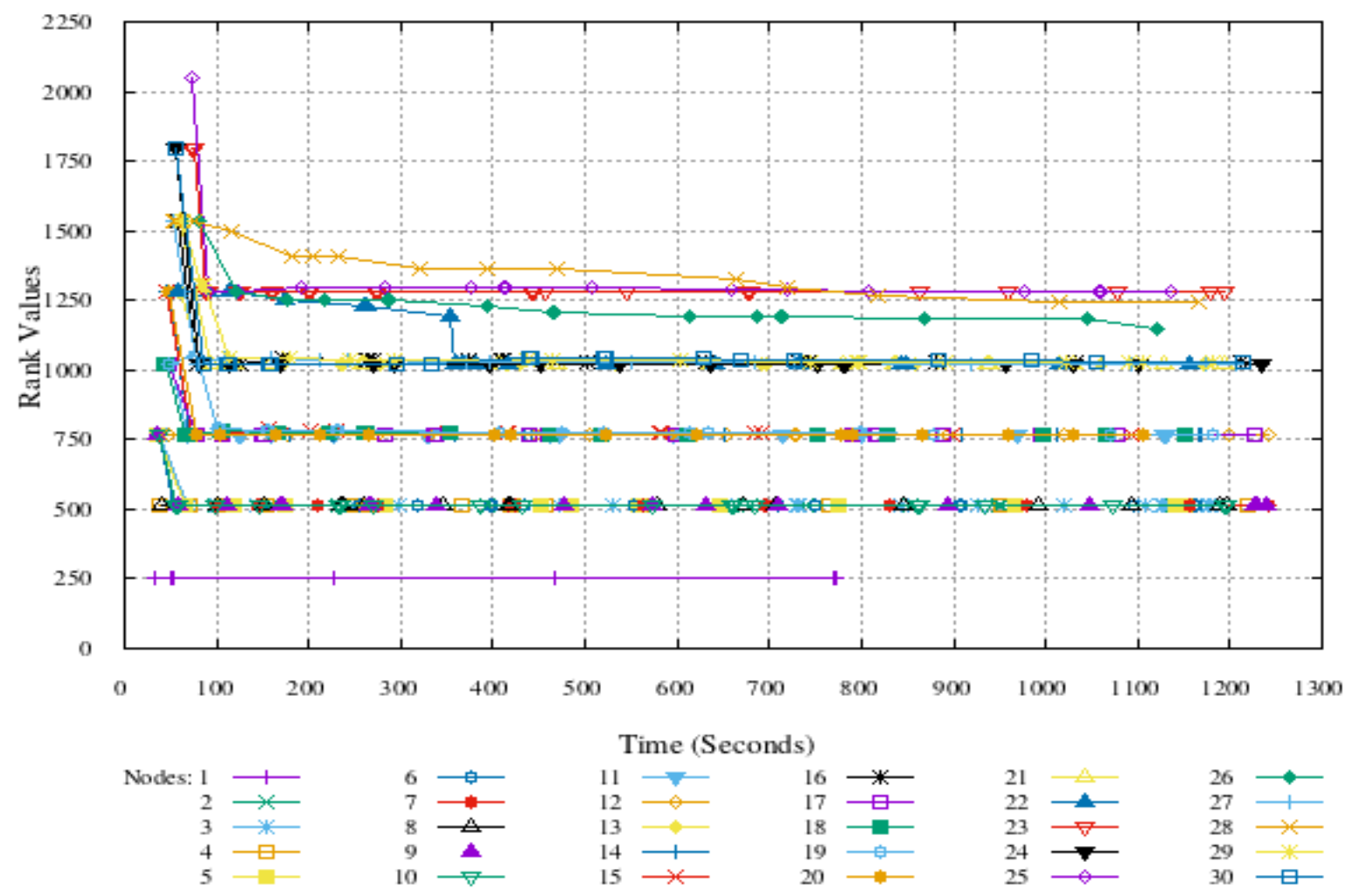

Figure 8 . The Rank values versus time for 30 motes

\subsection{Second scenario}

The Figure 9 depicts the motes and ETX values, mote number 1 (sink) acts as a root and the remaining motes form the Destination Oriented DAGs (DODAGs), due to the ETX calculation each node selects it is preferred parent, therefore it is obvious that all motes are contained in path oriented toward.

The motes are organized into three level as follows, level one include motes numbers $(33,34,38,39)$ terminating at three motes (roots), level two include motes numbers $(2,3,4,5,6,9,10,11,12,13,14,15$, $16,20,22,24$ ) terminating at two motes (roots) and level three include the other motes terminating at mote number 1 (sink root), as a result the DODAGs rooted at a single DAG root mote number 1 to form a diagram look like the tree, there is no cycles exist.

The RPL sends DODAG Information Solicitation (DIS) messages to scan the entire candidate neighbours' motes, as it has shown in Figure 10 below

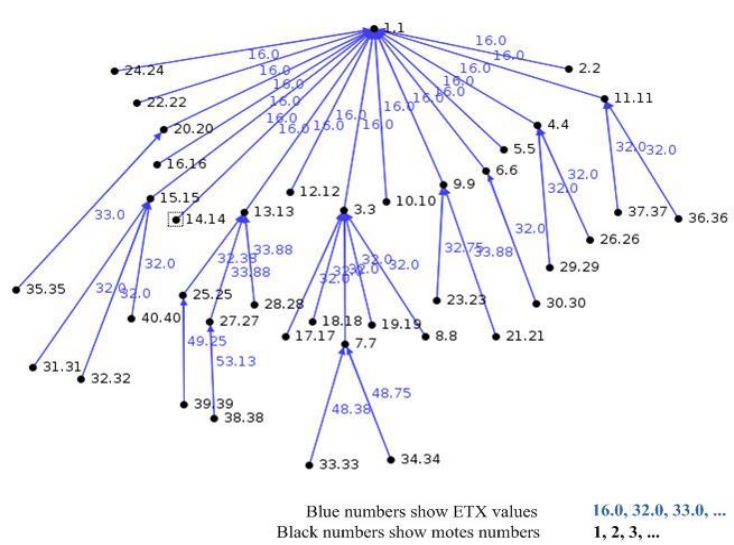

Figure 9. The RPL motes and ETX values

the numbers of the candidate neighbours for each mote with respect to the time, therefore the numbers of the neighbour's motes increase as far as all the 
motes that are qualified potentially to become a parent discovered, so, the obtained list of neighbours can be use by the OF0 in the selection procedures of the preferred parent.

The Figure 11 shows the ETX values versus the time for all motes start at time $=20: 24$ to reflect the path (link) quality, most of the motes that are in the transmission range are connected directly to the root (sink) and each one have got fixed ETX value equal to 16 , in contrast with the motes that are far away from the transmission range we find the motes have different values of ETX as they have shown in the figure, e.g. mote such as number 39 at time 20:24 the $\mathrm{ETX}=65$, and it is obviously this value decreased until it reach 49.25 , because the ETX values had been updating along the path.

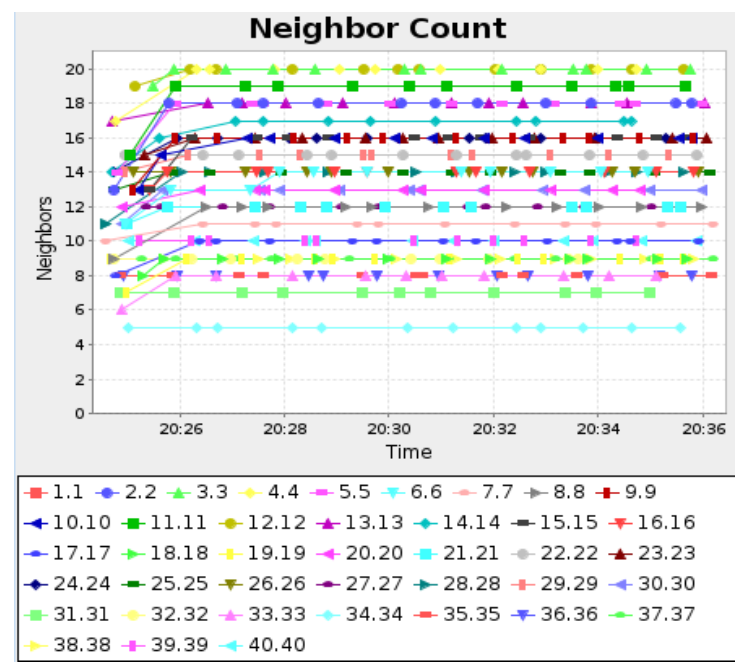

Figure 10. Neighbours numbers for each motes

As consequence the mote updates the ETX value and advertises the new path ETX by DODAG information object (DIO) messages when it selects any mote as it is preferred parent.

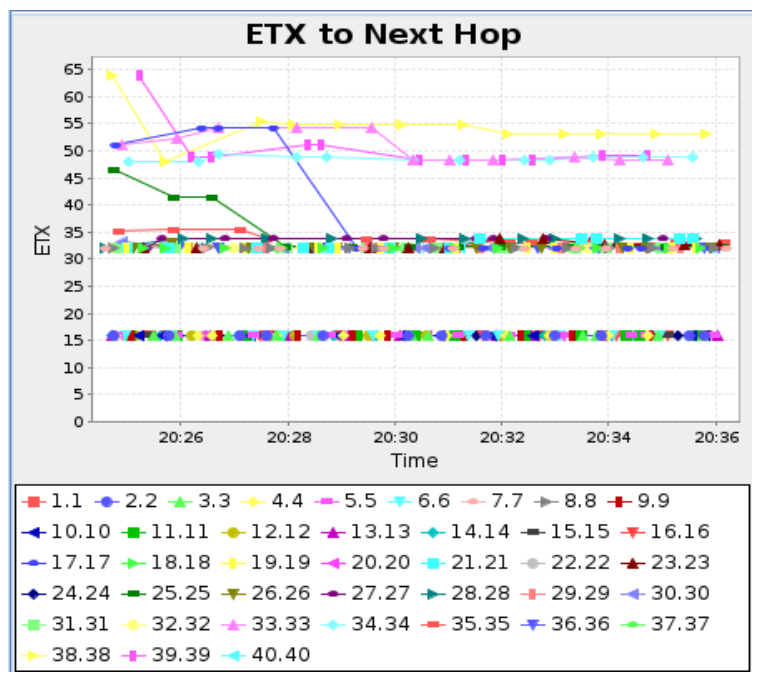

Figure 11. The ETX values versus the time
There are many motes have changed the number of the traverse motes from one to another but did not reduce the number of the routers to the sink, the change it was made according to the ETX values, except the mote number 17 it reduced the number of the traversed motes from three to two and chose the good link quality (ETX values).

The Figure 12 shows the RDC observation the listen time for the packet transmission from the neighbours' motes and transmission time, so the sender repeatedly sends the data packets until it receives an ACK from the receiver which has effects in transmission ratios differ from the motes inside the sink transmission range and the outside the sink transmission range. Also, ETX has effect in the RDC as we can see in Figure 11 the mote number 34 approximately has fixed value of ETX, therefore it take less duty cycle in contrast with other motes that fall in the same radio range.

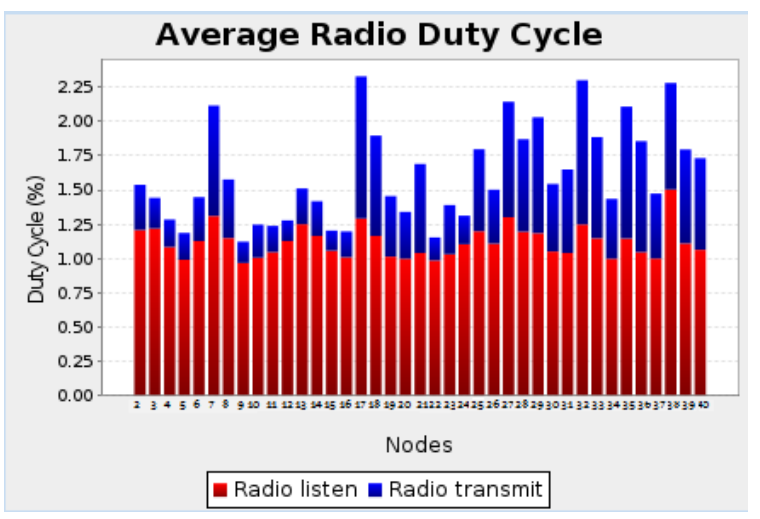

Figure 12. Average radio duty cycle

The Figure 13 represents the average power consumption, so the consumed powers by the motes in the communication procedures (listen, transmit) it is more than the power that consumed in the computation activity by the CPU, moreover the most energy that is consumed by the motes it is occurs during the listen period.

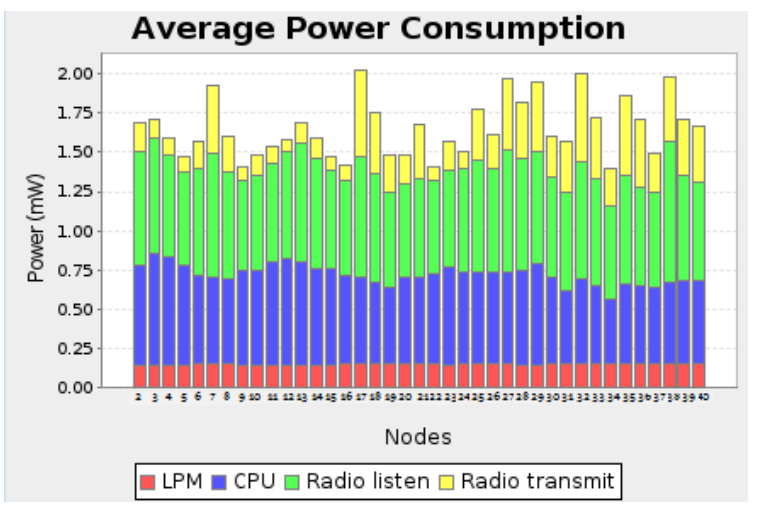

Figure 13. Average power consumption

From Figures 12 and 13 we observe the motes that 
have the shortest duty cycle values it also consumed the shortest energy values and vice versa.

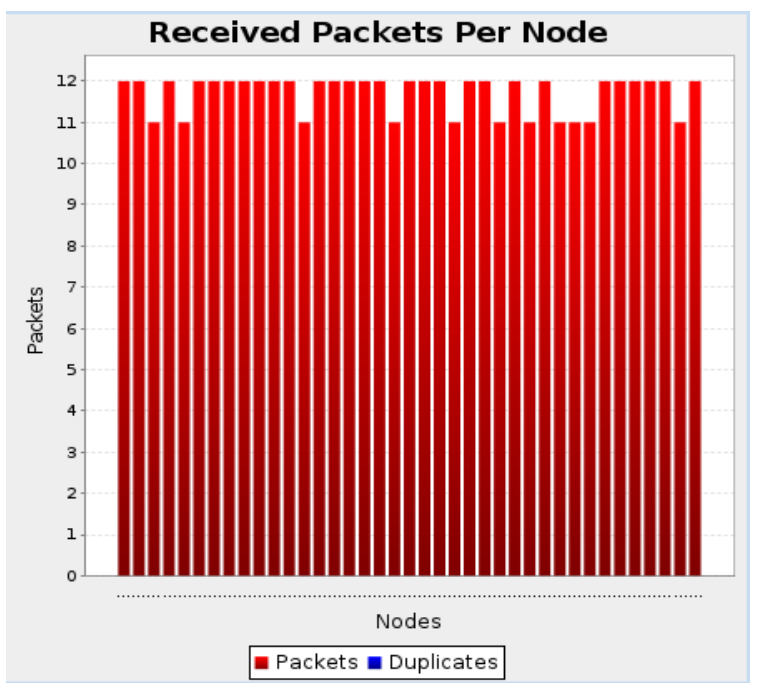

Figure 14. The numbers of the received packets

The Figure 14 represents the numbers of the received packets by the motes it is vary between 11 and 12 packets, the total number of the received packets equal 457 and the number of the lost packets estimated equal zero lost packets, this is mean the RPL shows good performance after it perform the DODAG routing environments.

\section{Conclusion}

The IPv6 Routing Protocol for Low-Power and Lossy Networks (RPL) introduced by The Routing Over Low-power Lossy (ROLL) networks, to support Low-power Lossy Networks (LLNs) which it always consist of numerous constrained devices, such networks include home automation application that contain constraint devices having slow CPU and small memory, as a result its put limit to the physical size and capacity of the battery; as well as in the industrial application the low-power device can improve the productivity, safety and efficiency of the plant worker; in this paper we provide a comprehensive analysis to the RPL and DODAGs message ( DIS, DIO, DAO) and represent their impacts on construction of RPL Instance, also we analyze the Rank computation that determine the stability and consistency of the routing topology, which is carrying by the DIOs message that is generated periodically by the trickle timer, as well as we evaluate the Expected Transmission Count (ETX), Radio Duty Cycle (RDC), energy consumption which it is a critical issue in LLNs, as a consequence we found its obviously the RPL is a good candidate to solve the routing issues over the constraint networks.

\section{References}

[1] Contikios, Contiki: The Open Source OS for the Internet of Things, Contiki OS. [Online]. Available: http://www.contikios.org/index.html, (Access Date: 23 January 2018).

[2] Moteiv Corporation, http://www.moteiv.com, (Access Date: 30 January, 2018).

[3] M.King and B. Zhu., (2006). "Tmote Sky: Datasheet," Moteiv Corporation, 11,13.

[4] T. Winter, A. Brandt, J. Hui, R. Kelsey, P. Levis, K. Pister, R. Struik, JP. Vasseur, R. Alexander and P. Thubert., (2012). RPL: IPv6 Routing Protocol for Low-Power and Lossy Networks, IETF, March.

[5] P. Levis, T. Clausen, J. Hui,O. Gnawali, J. Ko., (2011). "The Trickle Algorithm," RFC 6206, March.

[6] K. Pister, N. Dejean, D. Barthel., (2012). "Routing Metrics Used for Path Calculation in Low-Power and Lossy Networks,” RFC 6551, March.

[7] P. Levis, A. Tavakoli, S., (2009). Dawson-Haggerty, "Overview of Existing Routing Protocols for Low Power and Lossy Networks," IETF, Apr 15,

[8] T.Zhang, X.Li., (2014). "Evaluating and Analyzing the Performance of RPL in Contiki," ACM, August 11.

[9] P. Thubert, (2012). Objective Function Zero for the Routing Protocol for Low-Power and Lossy Networks (RPL), RFC 6552, March.

[10] O.Gnawali, P. Levis., (2012). The Minimum Rank with Hysteresis Objective Function, RFC 6719, September.

[11] S.Deering, R. Hinden., (1998). Internet Protocol, Version 6 (IPv6) Specification, RFC 2460, December.

[12] K.Pister, P.Thubert, S. Dwars, T. Phinney., (2009). Industrial Routing Requirements in Low-Power and Lossy Networks, RFC5673, October.

[13] A. Brandt, J. Buron, G. Porcu., (2010). "Home Automation Routing Requirements in Low-Power and Lossy Networks," RFC5826, April.

[14] J. Hui, P. Thubert, "Compression Format for IPv6 Datagrams over IEEE 802.15.4-Based Networks", RFC 6282, RFC6282, September 2011.

[15] P. Thubert, C. Bormann, L. Toutain, R. Cragie., (2017) "IPv6 over Low-Power Wireless Personal Area Network (6LoWPAN) Routing Header,” RFC8138, April 2017.

[16] IEEE, (2011). 802.15.4-2011 IEEE Standard for Local and metropolitan area networks Part 15.4: Low-Rate Wireless Personal Area Networks (LR-WPANs).

[17] K. Mohamed, A. Abdelrahim., (2019). Implementation and Analysis of the 6LoWPAN for the Internet of Things Applications: Future Networks," International Journal of Computer Science and Information Security (IJCSIS), Vol. 17, No. 4, April. 
[18] K. Mohamed, S. Hussein, A. Abdi, A. El Seddiq., (2018). Studying the TCP Flow and Congestion Control Mechanisms Impact on Internet Environment, International Journal of Computer Science and Information Security (IJCSIS), Vol. 16, No. 11, November.

[19] O. Iova, G. Picco, T. Istomin, C. Kiraly., (2016). "RPL, the Routing Standard for the Internet of Things Or Is It?, IEEE Communications Magazine, Institute of Electrical and Electronics Engineers.

[20] J. Ko, A.Terzis, S.Haggerty, D.E. Culler, J.W. Hui, P.Levis., (2011). "Connecting Low-Power and Lossy Networks to the Internet," IEEE Communications Magazine, April.

[21] M. Del., (1981). Transmission Control Protocol, RFC 793 September.

[22] J. Postel., (1980). User Datagram Protocol”, RFC 768, August.

[23] A. Dunkels., (2011). The contikimac radio duty cycling protocol, In Swedish Institute of Computer Science, (SICS) Technical Report T2011:13, December.

[24] M.Michel and B.Quoitin, "Technical Report: ContikiMAC performance analysis," arXivLabs, (Access Date: April, 2016).

[25] Wireshark Foundation, "Wireshark, Available: http://www.wireshark.org/ (Access Date: 12 March, 2019).

List of Abbreviations and Acronyms

6LowPAN IPv6 over low power Wireless Personal Area network

DAG Directed Acyclic Graph

DAO Destination Advertisement Object

DIO DODAG Information Object

DIS DODAG Information Solicitation

DODAG Destination Oriented DAG

ETX Expected Transmission Count

IEEE Institute of Electrical and Electronic Engineers

IETF Internet Engineering Task Force

IOTs Internet of Things

LLNs low-powered lossy Networks

MRHOF Minimum Rank with Hysteresis Objective

Function

OF0 Objective Function Zero

RDC Radio Duty Cycle

ROLL Routing Over Low-power and Lossy-network

RPL IPv6 Routing Protocol for Low-Power and

Lossy Networks

WSN Wireless Sensors Networks 